\title{
Commitment Devices as Behavior Change Support Systems: A Study of Users' Perceived Competence and Continuance Intention
}

\author{
Michael Oduor and Harri Oinas-Kukkonen \\ Oulu Advanced Research on Services and Information Systems \\ P.O. Box 3000, FI-90014 University of Oulu, Finland \\ \{michael.oduor, harri.oinas-kukkonen\}@oulu.fi
}

\begin{abstract}
The design principles of persuasive systems and the corresponding software features have been shown to have a positive effect on individuals' behavior and systems use. This study continues along the same line by analyzing the effects of these design principles on users' perceived competence and intention to continue to use an online commitment device. A structural equation modelling approach is used to identify the factors that affect the intention to continue to use the system. Data $(N=173)$ collected from the system's users is tested against the proposed research model. The results show that $37 \%$ of users' continuance intention is explained by the implemented persuasive software feature categories (computer-human dialogue support, primary task support, perceived credibility, and social support) and perceived competence. Of these categories, primary task support has the strongest effect on perceived competence and continuance intention. The study concludes with a discussion and recommendations for future research.
\end{abstract}

Keywords: persuasive systems design, continuance intention, perceived competence, behavior change, commitment device

\section{$1 \quad$ Introduction}

People require self-commitment devices because individuals set goals with varying degrees of formality that in most cases the individuals fail to meet primarily due to a lack of self-control. This draws into doubt the standard model of human behavior: that people are rational beings who when given a set of options always choose the most beneficial one [1]. There are numerous examples of suboptimal health behavior, for example, unwillingness to think about problems when risks are known or data are ambiguous, in which people do not follow through on their (behavior change) goals or make self-beneficial decisions [2].

Failing to meet goals can lead to entering into a commitment contract, a form of a commitment device that is an actual contract between two parties, rather than a unilateral arrangement employed by an individual to restrict his or her own choices [1]. A commitment contract is based on understanding attitude-behavior inconsistency 
(not doing what one has stated one will do or not acting according to one's own beliefs) and how incentives get people to work. As the role of information technology increases in people's day-to-day activities, decision making, and experiences, new opportunities to assist people in making self-beneficial choices have arisen through, for example, the use of persuasive systems to digitally present choices in a way that leverages people's decision-making processes, thus encouraging them to make selfbeneficial decisions $[3,4]$.

Persuasive systems are interactive systems that motivate users and are designed with the intent to change users' attitudes and/or behavior without coercion or deception [3]. These systems and technology, in general, are more effective in persuasion and offer a potential path to long-term behavior change as they leverage technology's capabilities [5] to influence users.

The objective of the present study, thus, is to investigate users' perceptions of an online commitment device. Specifically, the study examines the effect of the persuasive system design (PSD) model's principles [3] on users' perceived competence and how this, in turn, predicts their intention to continue using the commitment device. Data $(N=173)$ collected from the system's users is tested against the proposed research model using partial least squares (PLS), a structural equation modeling approach. Results show that primary task support from the PSD software feature categories is the strongest predictor of users' perceived competence and continuance intention.

\section{Theoretical Background}

The underlying principle behind persuasive systems is understanding users and integrating this understanding into developing systems (or implementing system features) that encourage users to more actively pursue or focus on their objectives. This could be through designing strategies that help people who want to change their everyday behavior [6], investigating how the design of persuasive systems and intervention characteristics influence users' adoption, continuance intention, and adherence to these systems [7-9], analyzing how the features implemented in a gamified persuasive system are compatible with users' goals and affect system usage, and whether this leads to the desired behavioral outcome [10], among many others.

Many people are aware of the changes they need to make in their behavior but usually have a problem following through on their intentions primarily due to a lack of self-control $[11,12]$. That is, people intend to make choices that carefully consider short-term and long-term cost and benefits, "but at the decision-making moment, they place disproportionate weight on immediate costs and benefits" [12]. Furthermore, some of the solutions people choose to help them change a behavior or form habits are often easy and do not have an impact because there are no hard commitments. A solution to this problem is the use of commitment devices: "a way of changing one's incentives to make an otherwise empty threat or promise credible" [11] and entering into an arrangement that restricts their future choice set by making some choices more expensive or making a present intention for a future action more substantial $[1,13]$. 
As commitment devices (see, e.g., [11] for a list of online commitment devices) also involve persuasive design to motivate users into action, it is essential to empirically analyze the persuasive elements in these systems.

\section{$3 \quad$ Research Model}

The PSD model [3] is used as the main conceptual framework in the present study. The model is an integrative framework encompassing the psychological principles behind the design of persuasive systems, the importance of considering the context of use, and the specific features implemented in persuasive systems [3]. In addition to the persuasive features, the study investigated the effect of perceived competence $[14$, 15] on continuance intention $[9,6]$. The measurement items for the constructs can be found in Appendix A. Figure 1 presents the research model.

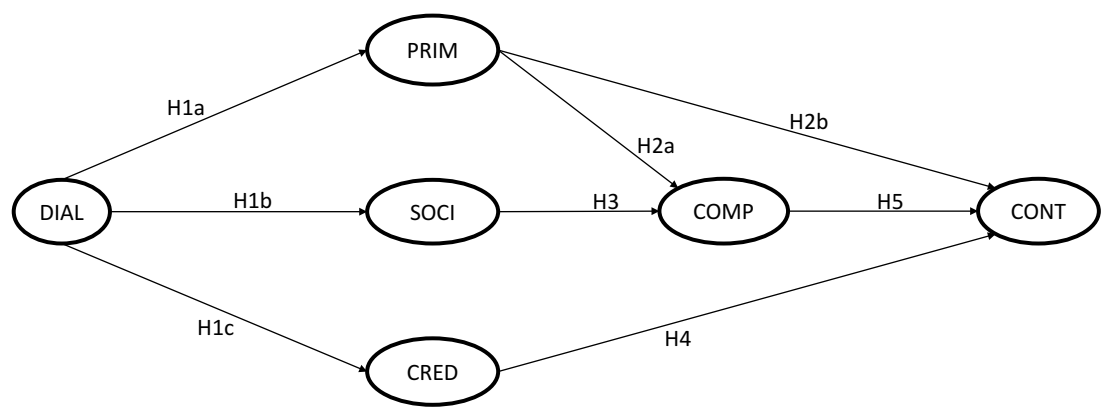

Fig. 1. Research Model

Note. DIAL $=$ Dialogue support; PRIM $=$ Primary task support; SOCI $=$ Social support CRED $=$ Perceived credibility COMP $=$ Perceived competence CONT $=$ Continuance intention.

Computer-human dialogue support. Computer-human dialogue support (henceforth, dialogue support) features facilitate and improve communication between a system and its users, especially in terms of feedback for better guiding users toward their intended goals $[3,17,18]$. Reminding users of their target behavior, for example, will more likely lead to goal achievement, and reminders in various forms [19], such as automated and personalized e-mails and phone calls triggered by technology or a clinician, have been shown to help in task completion $[20,21]$. Thus, these features are strongly linked to primary task support. Individuals' interactions with computers are also fundamentally social and from a user's perspective, computers can, in addition to being tools and mediums that increase capability and provide interactive experiences, be social actors that create and enhance relationships [5, 22]. Dialogue support also affects users' positive feelings toward the system, which likely influences the individuals' confidence (credibility) [8]. Therefore, we propose the following hypotheses: 
H1a: Dialogue support positively affects primary task support.

H1b: Dialogue support positively affects social support.

H1c: Dialogue support positively affects perceived credibility.

Primary task support. Primary task support refers to the way in which a system helps users carry out their primary task (that is, the real-world activity that they would like to complete) [3]. Primary task support aims to enhance users' self-efficacy and reduce the cognitive burden associated with using information systems by providing examples of the correct behavior, enabling reflection, goal-setting, and tracking of one's progress $[8,9]$. Therefore, it is likely that the more a system supports users in meeting their primary objective, the more they will feel competent and continue to use the system. We propose the following:

H2a: Primary task support positively affects perceived competence.

$\mathrm{H} 2 \mathrm{~b}$ : Primary task support positively affects continuance intention.

Social support. Social support features motivate users by leveraging social influence, especially as social relationships are increasingly maintained and nurtured through computer-mediated communications [3,9]. For this study, a modification of one indicator from social learning, another from social cooperation [23], and a selfdeveloped item were used to measure social support. Adding social elements can have a positive effect on a target behavior and enhance the effectiveness of a persuasive system [20]. Social support is also an important antecedent to self-efficacy; therefore, individuals who perceive they have received more social support are likely to have higher self-efficacy beliefs, which has a positive influence on the individuals' competence $[24,25]$. Thus, we hypothesize:

H3: Social support positively affects perceived competence.

Perceived credibility. Perceived credibility deals with designing credible and subsequently more persuasive systems. Perceived credibility comprises trustworthiness, expertise, surface credibility, real-world feel, authority, third-party endorsements, and verifiability [3]. Research has shown that understanding how users perceive the credibility of reviews, for example, is important for the survival and development of online discussion forums [26]. Cheung et al. [26] found that a high level of electronic wordof-mouth review credibility enhances users' adoption desires for online recommendations, ultimately leads to continuous use, and attracts repeat visits to other consumer recommendations forums [26]. Therefore, we propose the following:

H4: Perceived credibility positively affects continuance intention.

Perceived competence. Competence is the desire to feel a sense of satisfaction and effectiveness in attaining important results [15]. Perceived competence is one of the three fundamental needs proposed in the self-determination theory [15]. The theory focuses on motivation and proposes that human beings have basic psychological 
needs for autonomy, competence, and relatedness [15]. Perceptions of competence are theorized to be important because they facilitate people's goal attainment and provide them with a sense of need satisfaction from engaging in an activity at which they feel effective [14]. Therefore, the more competent one feels, the more likely one is to continue with an activity. Consequently, we propose:

H5: Perceived competence positively affects continuance intention.

\section{$4 \quad$ Research Methodology}

Study context. The artifact examined in the present study is a Web-based Quantified Self (self-tracking data collection and visualization) with commitment contracts system $^{1}$. In the application, an individual creates a contract to spend either less or more time on a particular activity, and if the individual does not stick to the goal(s), then he or she is charged. The system adds real consequences for not meeting planned goals. An individual can set a goal (lose weight, exercise, to-do tasks, etc.), manually input the required results, or connect to external services for automatic reporting. Progress is plotted on a graph that is easily accessible from one's account either through mobile devices or online. If the individual stays on track, then the service is free; however, if the individual goes off track, then he or she pledges money to continue progressing with his or her goal. If the individual goes off track again, he or she is charged.

Data collection and respondent characteristics. Between March and May 2016, an online survey of the system users was conducted. Data were collected using an online software tool (Webropol) over a 7-week period. Participants were recruited through a public link that was sent to their email addresses. The survey had two main parts. The first part consisted of demographic questions and questions related to the main goal tracked, use history, and use frequency. A total of 173 responses without missing data were collected. Overall, about $76 \%$ of the respondents were male, $50 \%$ were 30 years old or younger, about $70 \%$ were either married or in a relationship, and $86 \%$ had at least an undergraduate degree. Tables 1 and 2 present the respondents' characteristics.

The second part was the main survey instrument (Appendix A) consisting of sevenpoint Likert scale items (ranging from strongly disagree to strongly agree).

\section{$5 \quad$ Results and Analysis}

The research model was analyzed using the structural equation modeling (SEM) approach, namely, PLS, a technique for simultaneously estimating relations among multiple constructs. SmartPLS software [27] was used for the data analysis. PLS-SEM analysis is composed of two steps: The first step assesses the measurement model, that is, analyzed the relation of each indicator with its corresponding constructs. In the research model, all of the constructs were reflective and measured with at least two

1 https://www.beeminder.com/ 
indicators. Reflective measurement models are typically assessed in terms of their internal consistency, reliability, and validity. If the measurement model meets the specified criteria, then the second step, the structural model, evaluates the hypothesized relations between the constructs. The evaluation of the predictive capabilities of the structural model is based on the significance of the path coefficients and the relations between the constructs [28].

\subsection{Measurement Model}

The measurement model represents the relation between constructs and their corresponding measures. Constructs' properties are assessed in terms of their validity and reliability, and measurement items that are not at acceptable levels are removed. Evaluating the measurement model addresses internal consistency (the composite reliability), indicator reliability, convergent validity, and discriminant validity. Reliability and consistency are measured using Cronbach's alpha (CA) and composite reliability $(\mathrm{CR})^{2}$ with values ranging between 0 and 1 . High values indicate higher levels of reliability, and values higher than the typically applied threshold of 0.7 are acceptable [29]. For composite reliability, values from 0.6 to 0.7 are acceptable in exploratory research, while in more advanced stages of research, values between 0.7 and 0.9 are considered satisfactory. A value of 0.95 , however, indicates unnecessary redundancy in the construct items [28], which was the case in the perceived credibility construct. Therefore, CRED 2 (The system provides believable content) was omitted from the construct to decrease the composite reliability to below 0.95 .

The inter-construct correlations and the square root of the average variance extracted (AVE) show that all the constructs share more variance with their indicators than with other constructs. This, in addition to the indicators' factor loadings, is a good measure of convergent validity - the extent to which two or more items measure the same construct [30]. High loadings indicate that the measurement items correctly measure the same phenomenon and share a high proportion of variance [28]. Items with outer loadings below 0.6 were omitted (in primary task support and dialogue support). The AVE values for all the constructs were above the suggested minimum of 0.5 [29]. Table 3 shows that all the measurement model criteria were met.

\subsection{Structural Model}

Assessing the structural model determines how well the empirical data support the theory, showing the relations between different constructs and specifying how the constructs are related [28]. The key results are the path coefficients and the $\mathrm{R}^{2}$ values, which represent the hypothesized relations among the constructs and the percentage of the total variance of the dependent variable explained by the independent variables. The complete bootstrapping method with 5,000 resamples and parallel processing with no sign changes was used. Two-tailed bias-corrected and accelerated (Bca) boot-

\footnotetext{
${ }^{2} \mathrm{CA}$ and $\mathrm{CR}$ are not commonly used abbreviations for Cronbach's alpha and composite reliability but due to space constraints the abbreviations are used in table 4 .
} 
strap was the confidence interval method used. Figure 2 presents the results of the path model analysis.

Table 1. Respondent characteristics $(N=173)$

\begin{tabular}{llll}
\hline Demographics & Value & Frequency & Percent (\%) \\
\hline Gender & Male & 131 & 75.7 \\
& Female & 39 & 22.5 \\
\multirow{5}{*}{ Age } & Unspecified & 3 & 1.7 \\
& 30 or under & 87 & 50.3 \\
& $31-40$ & 53 & 30.6 \\
Marital status & M1-50 & 22 & 12.7 \\
& 50 and older & 11 & 6.4 \\
& Married & 58 & 33.5 \\
& In a relationship & 62 & 35.8 \\
& Single & 53 & 30.6 \\
& High school & 20 & 11.6 \\
& Vocational training & 4 & 2.3 \\
& Bachelor's degree & 66 & 38.2 \\
& Master's degree & 49 & 28.3 \\
& Other advanced degree & 9 & 5.2 \\
& Doctoral degree & 25 & 14.5 \\
\hline
\end{tabular}

Table 2. System version, main goals tracked, and use history

\begin{tabular}{llll}
\hline & Value & Frequency & Percent (\%) \\
\hline Version & Free Plan & 128 & 74 \\
& Bee Lite & 11 & 6.4 \\
& Plan Bee & 27 & 15.6 \\
Goals & Beemium & 7 & 4.0 \\
& Fitness and Training & 34 & 19.7 \\
& Health & 38 & 22 \\
& Productivity & 80 & 46.2 \\
Use history & Learning & 21 & 12.1 \\
& 6 months or less & 40 & 23.1 \\
& 6 months to a year & 23 & 13.3 \\
& 1 to 2 years & 35 & 20.2 \\
& 2 to 3 years & 33 & 19.1 \\
& 3 or more years & 42 & 24.3 \\
\hline
\end{tabular}


Table 3. Reliability and validity of the constructs

\begin{tabular}{lll|l|llllll}
\hline & CA & CR & AVE & COMP & CONT & CRED & DIAL & PRIM & SOCI \\
\hline COMP & 0.88 & 0.916 & 0.73 & $\mathbf{0 . 8 5 5}$ & & & & & \\
CONT & 0.91 & 0.938 & 0.79 & 0.286 & $\mathbf{0 . 8 9 0}$ & & & & \\
CRED & 0.91 & 0.945 & 0.85 & 0.052 & 0.137 & $\mathbf{0 . 9 2 2}$ & & & \\
DIAL & 0.72 & 0.841 & 0.63 & 0.134 & 0.241 & 0.266 & $\mathbf{0 . 7 9 8}$ & & \\
PRIM & 0.72 & 0.838 & 0.64 & 0.348 & 0.603 & 0.268 & 0.377 & $\mathbf{0 . 8 0 0}$ & \\
SOCI & 0.74 & 0.848 & 0.65 & 0.074 & 0.190 & 0.154 & 0.301 & 0.178 & $\mathbf{0 . 8 0 7}$ \\
\hline
\end{tabular}

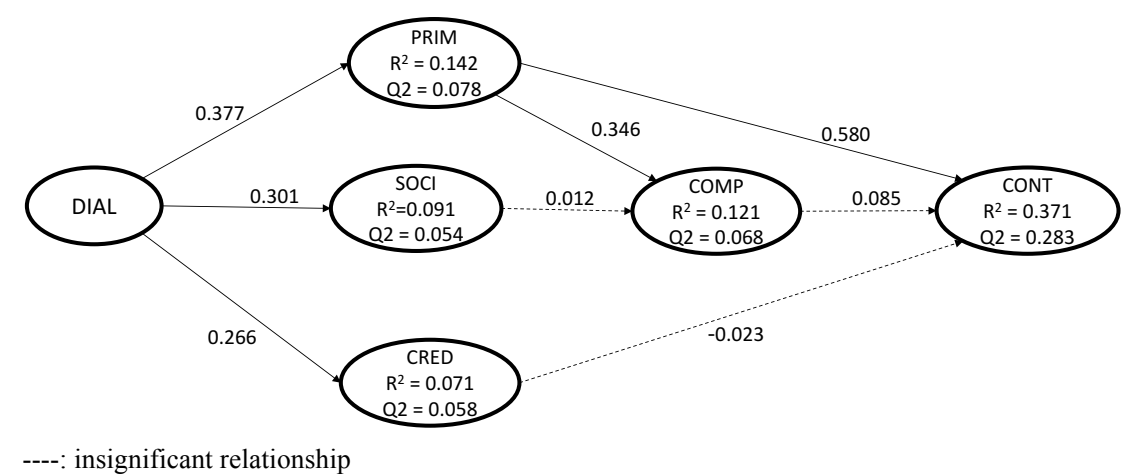

Fig. 2. Structural model results for the full sample

The results of the PLS analysis (Fig 2) provide moderate support for the research model. Five (H1a, H1b, H1c, H2a, H2b) of the eight hypotheses were supported at $\mathrm{p}<$ .001 , and primary task support and social support explain $12 \%$ of the variance in perceived competence. Dialogue support explained $14.2 \%$ of the variance in primary task support, $9 \%$ of the variance in social support, and $7 \%$ of the variance in perceived credibility. A substantial amount of the variance $(37 \%)$ in intention to continue using the system was explained by primary task support, whereas perceived competence and perceived credibility were found not to be strong predictors of intention to continue to use the system.

Table 4 shows the total effects (direct and indirect effects) and the effect sizes of each construct on the corresponding dependent variables' variance. Effect sizes determine whether the effects shown by path coefficients are small (.02), medium (0.15), or large (.35) [31]. Effect sizes below .02 are considered too weak to be relevant, which is the case for three hypotheses. Dialogue support has the highest total effect (with a medium effect size) on primary task support, whereas primary task support has the highest total effect on perceived competence (a medium effect size) and continuance intention (a large effect size). Dialogue support also has an indirect positive effect on perceived competence and continuance intention. 
Table 4. Total effects and effect sizes (Cohen's $f^{2}$ in parentheses)

\begin{tabular}{lllllll}
\hline & COMP & CONT & CRED & DIAL & PRIM & SOCI \\
\hline COMP & & 0.085 & & & & \\
& & $(0.01)$ & & & & \\
CRED & & -0.023 & & & & \\
& & $(0.001)$ & & 0.377 & 0.301 \\
DIAL & 0.134 & 0.224 & 0.266 & & $(0.166)$ & $(0.100)$ \\
& & & $(0.076)$ & & \\
PRIM & 0.346 & 0.609 & & & & \\
& $(0.132)$ & $(0.436)$ & & & & \\
SOCI & 0.012 & 0.001 & & & & \\
\hline
\end{tabular}

The predictive relevance of the model was also assessed using Stone-Geisser's crossvalidated redundancy measure (the $\mathrm{Q}^{2}$ value). See Table 5. Values above zero for a certain reflective endogenous construct indicate the path model's predictive relevance for that particular construct [28]. Predictive relevance was demonstrated for all endogenous constructs, with continuance intention exhibiting moderately strong predictive validity.

Table 5. Predictive relevance $\left(Q^{2}\right.$ values $)$

\begin{tabular}{ccccc}
\hline COMP & CONT & CRED & PRIM & SOCI \\
\hline 0.068 & 0.283 & 0.058 & 0.078 & 0.054 \\
\hline
\end{tabular}

\section{Discussion}

The goal of the present study was to examine the applicability of PSD in explaining users' perceived competence and how PSD and competence influence intention to continue using an online commitment device. Continuance intention refers to users' intention to continue using a system after initial acceptance [16]. Continued use instead of initial acceptance explains the success of a system because the psychological motivations that affect users' continuance decisions emerge only after individuals have started using the system [16].

All relations (Fig. 2) except H4 (perceived credibility positively affects continuance intention) were found to be positively related. However, two of the positive relations (H3 and $\mathrm{H} 5)$ and $\mathrm{H} 4$ (which was negative) had very low path coefficients $(0.012,0.085$, and -0.023$)$ values meaning the practical significance of the associations is very low. These findings extend the literature on adoption and continuance intentions for persuasive systems [9, 10, 24] by identifying primary task support as an important predictor of perceived competence and continuance intention. This is consistent with findings in Lehto and Oinas-Kukkonen's [9] post-hoc analysis of simpler path models that test the effect of PSD features on use continuance.

The five positive relations (dialogue support predicts primary task support, social support, and perceived credibility, and primary task support predicts perceived com- 
petence and continuance intention) demonstrate the important role of PSD in use continuance. In line with previous studies $[8,20,22]$ that showed the importance of dialogue support, it was also shown in the present study to be an important factor in evaluating the effectiveness of a system. The latter two relations (primary task support as a predictor of competence and continuance) indicate that supporting a user's primary goal likely leads to him or her feeling more competent and increases the likelihood of continued use.

Interestingly, the direct effect between social support and perceived competence is weak, in contrast to that in [25], for example, who found that social support had an indirect total effect on users' nutrition through self-efficacy and self-regulation. This result may be due to the unique nature of the system compared with other persuasive systems and the social support construct itself. Two measurement items for the construct referred to the perception of support through the system, and the self-developed item was support mediated by the system (Appendix A). On the questionnaire, there was also a question asking users whether they had social supporters (self-appointed peers who can follow up on users' progress as this an optional feature in the system), and $91 \%(n=158)$ replied no. This can help explain the statistically insignificant relation between social support and perceived competence as not many of the respondents perceived or had this type of social support.

The relation between perceived competence and continuance intention, although positive, was statistically insignificant which warrants further investigation. A possibility, as it is one of the three psychological needs [15], is to extend the present model with these other psychological constructs, examine how they correlate with the PSD features, and affect continuance intention. The surprisingly negative but statistically insignificant relation between perceived credibility and continuance intention in contrast to Lehto and Oinas-Kukkonen [10] and Cheung et al. [26] also warrants further attention.

The study had several limitations. First, some of the relations in the model, even those shown to be strong in previous research, have lower or statistically insignificant values. Future research should examine the weaker links more closely. A second limitation is that the research model was applied to only one system. Thus, caution should be exercised about the generalization of the findings to other contexts. Nonetheless, the results should be applicable to other similarly designed systems or those with similar objectives to the one investigated. Last, the effects of demographic attributes and use characteristics were not analyzed in the present study. Future research may employ different procedures, such as subgroup analysis and application of constraints for data selection, to better explain the effects of the various PSD strategies on user behavior.

\section{Conclusion}

In summary, in accordance with previous research, this study provides evidence that persuasive system design features are associated to varying degrees with users' perceived competence and continuance intention of a commitment device. Further, as in 
several previous studies, dialogue support was found to have a crucial role in the effectiveness of the system as this feature strongly supports users' interactions with the system and facilitates completion of primary tasks. Primary task support also strongly predicts users' confidence in their ability to carry out a task and their intention to continue using a system. Understanding the place of persuasive systems design in the context of fulfilling users' needs during user engagement with the systems could provide a means to counterbalance factors that lead to the non-adoption or failure of digital interventions, especially considering the nature of commitment devices in which something (usually monetary) is at stake if specific objectives are not fulfilled. As the system investigated supported the pursuance of different goals, how carrying out these different goals is supported by PSD and how this can help in changing long-term behavior should be addressed in future research.

\section{Acknowledgments}

We would like to thank Daniel Reeves, the Co-founder/CEO of Beeminder for helping with the distribution of the survey to the system's users.

\section{References}

[1] G. Bryan, D. Karlan, and S. Nelson, "Commitment devices," Annu. Rev. Econ., vol. 2, no. 1, pp. 671-698, 2010.

[2] B. K. Mogler, S. B. Shu, C. R. Fox, N. J. Goldstein, R. G. Victor, J. J. Escarce, and M. F. Shapiro, "Using Insights From Behavioral Economics and Social Psychology to Help Patients Manage Chronic Diseases," J. Gen. Intern. Med., vol. 28, no. 5, pp. 711718, May 2013.

[3] H. Oinas-Kukkonen and M. Harjumaa, "Persuasive systems design: Key issues, process model, and system features," Commun. Assoc. Inf. Syst., vol. 24, no. 1, p. 28, 2009.

[4] M. K. Lee, S. Kiesler, and J. Forlizzi, "Mining Behavioral Economics to Design Persuasive Technology for Healthy Choices," in Proceedings of the SIGCHI Conference on Human Factors in Computing Systems, 2011, pp. 325-334.

[5] B. J. Fogg, "Persuasive Technology: Using Computers to Change What We Think and Do," Morgan Kauffman, 2003.

[6] S. Consolvo, D. W. McDonald, and J. A. Landay, "Theory-driven Design Strategies for Technologies That Support Behavior Change in Everyday Life," in Proceedings of the SIGCHI Conference on Human Factors in Computing Systems, 2009, pp. 405-414.

[7] S. M. Kelders, R. N. Kok, H. C. Ossebaard, and J. E. W. C. Van Gemert-Pijnen, "Persuasive system design does matter: a systematic review of adherence to web-based interventions.," J. Med. Internet Res., vol. 14, no. 6, p. e152, Jan. 2012.

[8] T. Lehto, H. Oinas-Kukkonen, T. Pätiälä, and O. Saarelma, "Consumers' Perceptions of a VirtuaL Health Check: An Empirical Investigation,” ECIS 2012 Proceedings. 2012.

[9] T. Lehto and H. Oinas-Kukkonen, "Explaining and predicting perceived effectiveness 
and use continuance intention of a behaviour change support system for weight loss," Behav. Inf. Technol., vol. 34, no. 2, pp. 176-189, Nov. 2013.

[10] C. Ebermann and B. Brauer, "The Role of Goal Frames Regarding the Impact of Gamified Persuasive Systems on Sustainable Mobility Behavior," ECIS 2016 Proceedings. 2016.

[11] D. Reeves, "How To Do What You Want: Akrasia and Self-Binding," Beeminder BLog, 2011. [Online]. Available: http://blog.beeminder.com/akrasia/. [Accessed: 10Feb-2016].

[12] J. L. Beshears, J. J. Choi, D. Laibson, B. C. Madrian, and J. Sakong, "Self control and liquidity: How to design a commitment contract,". RAND Working Paper Series WR895-SSA, 2011.

[13] N. Moraveji, R. Akasaka, R. Pea, and B. J. Fogg, "The Role of Commitment Devices and Self-shaping in Persuasive Technology," in CHI '11 Extended Abstracts on Human Factors in Computing Systems, 2011, pp. 1591-1596.

[14] Selfdeterminationtheory.org, "Perceived Competence Scales." [Online]. Available: http://selfdeterminationtheory.org/perceived-competence-scales/. [Accessed: 22-Sep2015].

[15] E. L. Deci and R. M. Ryan, "The" what' and" why' of goal pursuits: Human needs and the self-determination of behavior," Psychol. Inq., vol. 11, no. 4, pp. 227-268, 2000.

[16] A. Bhattacherjee, "Understanding Information Systems Continuance: An expectationconfirmation Model.," MIS Q., vol. 25, no. 3, pp. 351-370, Sep. 2001.

[17] T. Lehto and H. Oinas-Kukkonen, "Persuasive features in web-based alcohol and smoking interventions: a systematic review of the literature.," J. Med. Internet Res., vol. 13, no. 3, p. e46, Jan. 2011.

[18] S. Langrial, T. Lehto, H. Oinas-Kukkonen, M. Harjumaa, and P. Karppinen, "Native Mobile Applications For Personal Well-Being: A Persuasive Systems Design Evaluation," PACIS 2012 Proceedings. 2012.

[19] L. M. Ritterband, F. P. Thorndike, D. J. Cox, B. P. Kovatchev, and L. A. GonderFrederick, "A behavior change model for internet interventions.," Ann. Behav. Med., vol. 38, no. 1, pp. 18-27, Aug. 2009.

[20] M.-C. Chiu, S.-P. Chang, Y.-C. Chang, H.-H. Chu, C. C.-H. Chen, F.-H. Hsiao, and J.C. Ko, "Playful Bottle: A Mobile Social Persuasion System to Motivate Healthy Water Intake," in Proceedings of the 11th International Conference on Ubiquitous Computing, 2009, pp. 185-194.

[21] S. Langrial, H. Oinas-Kukkonen, P. Lappalainen, and R. Lappalainen, "Rehearsing to control depressive symptoms through a behavior change support system," in CHI'13 Extended Abstracts on Human Factors in Computing Systems, 2013, pp. 385-390.

[22] C. Nass and Y. Moon, "Machines and Mindlessness: Social Responses to Computers," J. Soc. Issues, vol. 56, no. 1, pp. 81-103, Jan. 2000.

[23] A. Stibe and H. Oinas-Kukkonen, "Using Social Influence for Motivating Customers to Generate and Share Feedback," in Persuasive Technology, Springer International Publishing, 2014, pp. 224-235.

[24] A. Bandura, "Health Promotion by Social Cognitive Means," Heal. Educ. Behav. , vol. 31, no. 2, pp. 143-164, Apr. 2004.

[25] E. Anderson, R. Winett, and J. Wojcik, "Self-regulation, self-efficacy, outcome expec- 
tations, and social support: Social cognitive theory and nutrition behavior," Ann. Behav. Med., vol. 34, no. 3, pp. 304-312, 2007.

[26] M. Y. Cheung, C. Luo, C. L. Sia, and H. Chen, "Credibility of Electronic Word-ofMouth: Informational and Normative Determinants of On-line Consumer Recommendations," Int. J. Electron. Commer., vol. 13, no. 4, pp. 9-38, Jul. 2009.

[27] C. M. Ringle, S. Wende, and Becker, "SmartPLS 3. Boenningstedt: SmartPLS GmbH," 2015. [Online]. Available: https://www.smartpls.com/.

[28] J. F. Hair Jr, G. T. M. Hult, C. Ringle, and M. Sarstedt, A primer on partial least squares structural equation modeling (PLS-SEM). Sage Publications, 2013.

[29] C. Fornell and D. F. Larcker, "Evaluating Structural Equation Models with Unobservable Variables and Measurement Error," J. Mark. Res., vol. 18, no. 1, pp. 39-50, 1981.

[30] R. P. Bagozzi and L. W. Phillips, "Representing and Testing Organizational Theories: A Holistic Construal," Adm. Sci. Q., vol. 27, no. 3, pp. 459-489, 1982.

[31] J. Cohen, Statistical Power Analysis for the Behavioral Sciences (2nd Edition). Routledge, 1988.

\begin{tabular}{l|l|l} 
Appendix A: & Measurement Instrument & Loadings \\
\hline Constructs & Indicators & 0.778 \\
Dialogue & The system rewards me & 0.787 \\
Support & The system provides me with appropriate feedback & $\mathbf{0 . 5 7 0}$ \\
& The system provides me with reminders for reaching my & \\
& goals & \\
& The system encourages me & 0.743 \\
\hline Primary & They system makes it easier for me to reach my goals & 0.825 \\
Task Support & The system helps me gradually reach my goals & 0.865 \\
& The system helps me keep track of my progress & 0.621 \\
& The system offers me personalized content* & $\mathbf{0 . 4 6 0}$ \\
\hline Perceived & The system provides trustworthy content & 0.949 \\
Credibility & The system provides believable content* & $\mathbf{0 . 9 6 1}$ \\
& The system provides accurate content & 0.954 \\
& The system provides professional information & 0.837 \\
\hline Social Support & The system enables me to share with others & 0.772 \\
& The system enables me to learn from others & 0.883 \\
& The system enables someone (chosen by me) to check on & 0.764 \\
& my commitments & \\
\hline Perceived & I feel confident in my ability to achieve my goal & 0.830 \\
Competence & I am capable of doing what it takes to achieve my goal & 0.899 \\
& I am able to achieve my goal & 0.803 \\
& I feel able to meet the challenge of fulfilling my goal & 0.885 \\
\hline Continuance & I intend to continue using the system & 0.943 \\
& I will be using the system in the future & 0.936 \\
& I am considering discontinuing using the system & 0.870 \\
& I am not going to use the system from now on & 0.805 \\
\hline & & \\
& &
\end{tabular}

*: removed items 